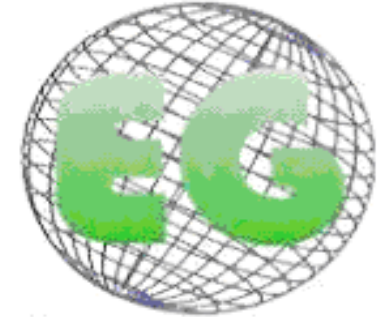

ISSN 1695-6141

$N^{\circ} 21$
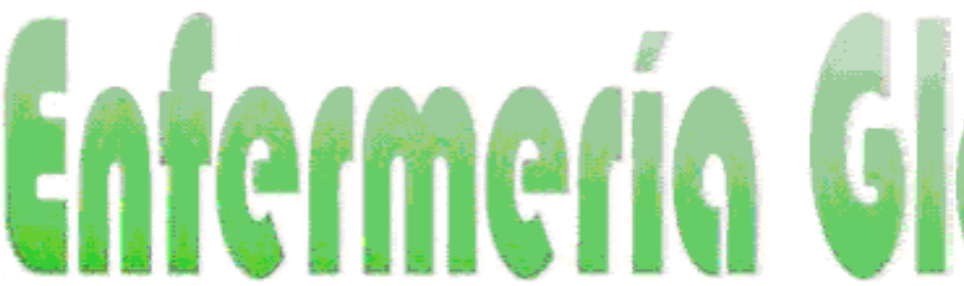

Revista electrónica trimestral de Enfermería

Enero 2011

www.um.es/egloball

\title{
LA VIOLENCIA CONTRA LAS MUJERES ENFERMERAS EN LA PAREJA. PRIMEROS RESULTADOS DE UN ESTUDIO EN ANDALUCÍA.
}

\author{
INTIMATE PARTNER VIOLENCE AGAINST FEMALE NURSES. FIRST RESULTS OF A \\ STUDY IN ANDALUSIA. \\ *Rodríguez Borrego, MA., "Vaquero Abellán, M., "Bertagnolli, L., "*Redondo \\ Pedraza, R., "* Muñoz Alonso, A. \\ "Escuela Universitaria de Enfermería de la Universidad de Córdoba. ** Distrito Sanitario Córdoba Centro.
}

\begin{abstract}
Palabras clave: Violencia de género. Violencia doméstica. Mujeres maltratadas. Violencia por compañero íntimo.

Keywords: Domestic violence. Battered women. Intimate partner violence (IPV
\end{abstract}

\section{RESUMEN}

Objetivo. Identificar violencia contra las mujeres enfermeras en la pareja a partir de una muestra de profesionales que prestan sus servicios en la Provincia de Córdoba.

Diseño. Estudio descriptivo transversal.

Emplazamiento. Hospitales y distritos sanitarios de la Provincia de Córdoba.

Participantes. Mujeres enfermeras que desarrollaban sus actividades profesionales en cualquiera de los sistemas de salud, público o privado, rural o urbano, en la Provincia de Córdoba.

Mediciones Principales. Características sociodemográficas y presencia de malos tratos (psíquico, físico y sexual), con el cuestionario validado por Delgado y colaboradores (2006).

Resultados. La Recodificación de las variables del maltrato más estricta muestra que el $47,1 \%$ del total de enfermeras en la muestra tuvo algún tipo de maltrato, del que el $41,2 \%$ fue exclusivamente psicológico. Una Recodificación del maltrato más permisiva indica que el $25,5 \%$ tuvo algún tipo de maltrato del que $19,6 \%$ fue psicológico. Considerando ambas se puede ver que el 2,9\% presentaron los tres tipos juntos (psicológico, físico y sexual) y psicológico acompañado del sexual; así como que el $11,8 \%$ presentó un maltrato más severo o de mayor gravedad.

Conclusiones. Establecida la existencia de malos tratos en la mujer enfermera se constata que las manifestaciones van referidas al aspecto psicológico.

\section{ABSTRACT}

Aim. To Identify intimate partner violence (IPV) against female nurses in a sample of nurses in Cordoba, Spain.

Design. Descriptive, cross-sectional study.

Setting. Hospitals and primary health care in Cordoba, Spain. 
Participants. One hundred and two female nurses working in urban or rural, public or private health centers in Cordoba, Spain.

Measures. Social-demographic characteristics and presence of abuse (psychological, physical and sexual).

Results. A) A stricter recoding of the parameters of abuse: overall, $47.1 \%$ had experienced some type of IPV, of which $41.2 \%$ was psychological. B) A more permissible recoding of the parameters of abuse: $25.5 \%$ had experienced some type of IPV, of which $19.6 \%$ was psychological. C) Both recodings: $2.9 \%$ had suffered three types of abuse together (psychological, physical and sexual) and both psychological and sexual IPV; likewise, 11,8\% reported more severe or more serious abuse.

Conclusion. The existence of intimate partner violence in female nurses was established and it was confirmed that this was expressed through psychological aspects.

\section{INTRODUCCIÓN}

Según las Naciones Unidas (1995) ${ }^{1}$ se entiende como violencia contra la mujer (VCM) todo acto de violencia sexista que tiene como resultado posible o real un daño físico, sexual o psíquico, incluidas las amenazas, la coerción o la privación arbitraria de libertad, ya sea que ocurra en la vida pública o en la privada. Actualmente constituye un grave problema de derechos humanos y salud pública que afecta a todos los sectores de la sociedad ${ }^{2,3,4}$, tanto por su incidencia como por su mortalidad ${ }^{5}$.

Una de las formas más frecuentes de violencia de género es la ejercida por el marido o compañero íntimo. Esta puede ser denominada violencia doméstica, violencia por compañero íntimo (VCI), violencia contra la mujer en la pareja o violencia conyugal ${ }^{6,7}$.

Las mujeres víctimas de violencia acuden más a los servicios sanitarios que las demás ${ }^{8,9}$. Estudios realizados en diversos países dan a conocer que un porcentaje importante de las mujeres que sufren violencia por su pareja acuden a los servicios de atención primaria ${ }^{8,9}$, salud mental ${ }^{10}$, traumatología $^{11}$ y urgencias $^{12,13}$.

No se puede ignorar que los problemas de salud derivados de la violencia doméstica no son reconocidos habitualmente por las/los profesionales sanitarios $8,9,14-16$.

Un alto porcentaje de los profesionales enfermeros son fundamentalmente mujeres, lo que ha llevado a cuestionarnos, si estas mujeres que entre sus responsabilidades profesionales tienen la competencia de detección, no estarán viviendo también situaciones de violencia de género por compañero íntimo. Por lo que hemos planteado un acercamiento al problema con un estudio a nivel de la Comunidad de Andalucía.

El trabajo que aquí se presenta corresponde a una muestra de profesionales que prestan sus servicios en la Provincia de Córdoba teniendo como objetivos identificar la existencia de malos tratos y describir las manifestaciones de $\mathrm{VCl}$ contra estas mujeres. A su vez se enmarca dentro de un proyecto más amplio en una red multinacional de trabajo en colaboración con Brasil, Ecuador, Portugal y Puerto Rico, que siguen la misma línea de investigación.

\section{MÉTODOS}

\section{Sujetos}

Estudio descriptivo transversal en el que se incluyeron enfermeras que desarrollaban sus actividades profesionales en los sistemas de salud, público o privado, rural o urbano, en la 
Provincia de Córdoba. Participaron tanto enfermeras base como las que ocupaban puesto de gestión.

\section{Muestra}

Para el cálculo de la muestra se utilizó una simulación que incluía: prevalencia de 12\% (tasa de maltrato a mujeres de la población general de España del año 1999) ${ }^{17}$, precisión de la estimación de $3 \%$ y un nivel de confianza de $95 \%$. Si bien el cálculo de la muestra correspondiente a Córdoba era de 49 sujetos, el número de cuestionarios distribuidos fue de 260 fundamentados en un escrupuloso respeto a la intimidad de las participantes, respeto que también nos aconsejó aleatorizar servicios asistenciales donde las enfermeras prestaban su servicios profesionales y no personas.

Finalmente en los cálculos estadísticos se incluyeron únicamente las enfermeras que habían tenido compañero íntimo actual o ex $(n=102)$.

\section{Ámbito}

Se contactó con un total de 11 centros sanitarios de Córdoba que correspondieron a 6 hospitales (4 públicos y 2 privados), 4 distritos sanitarios y 1 compañía privada, aceptando todos colaborar en el estudio. El periodo de recogida de los datos fue de mayo a julio de 2008.

\section{Instrumento de medición y variables estudiadas}

El instrumento utilizado para recoger la información fue un cuestionario estructurado autoadministrado creado por Delgado y colaboradores $(2006)^{18}$. Este consta de 10 preguntas tipo Likert, seis correspondientes al maltrato psíquico; tres, al maltrato físico y una, al maltrato sexual. Además, complementado con preguntas referentes a las características sociodemográficas. En la distribución del mismo se incluyó una carta explicativa del proyecto como forma de consentimiento informado en la que no se requería la firma de los participantes en el estudio para preservar el anonimato.

\section{Variables sociodemográficas}

Se contemplaron las variables cuantitativas: edad de la enfermera, años de relación con la pareja y número de personas dependientes; y las cualitativas: provincia de origen, estado civil, convivencia (pareja, hijos, familia origen, amigos), centro de trabajo, ámbito de trabajo, puesto de trabajo, sustento económico familiar (a cargo de la entrevistada o de su pareja), clase social de la pareja (según la sociedad Española de Epidemiología, 1995), hijos y/o personas dependientes.

\section{Variables del maltrato}

El cuestionario de Delgado y colaboradores $(2006)^{18}$ está formado por preguntas cerradas enfocadas a la detección de malos tratos físicos, psicológicos y sexuales; y con 5 posibles respuestas: nunca, casi nunca, algunas veces, bastantes veces y casi siempre.

Las variables fueron planteadas como dicotómica: "si", para la existencia de malos tratos y "no", para la ausencia de malos tratos. En la escala de medida se han utilizado dos perspectivas, una es la empleada por los autores del cuestionario y que utiliza un criterio más permisivo en algunas cuestiones de maltrato psicológico como «respetar los 
sentimientos», «insultar...» e «intentar controlar...» (tabla 1) y otra, la del grupo investigador, con un criterio más estricto, en cuanto a las preguntas mencionadas (tabla 2). Se contemplaron dos variables policotómicas: el tipo de maltrato (ningún tipo, psicológico, físico, sexual, psicológico y físico, psicológico y sexual, físico y sexual y psicológico, físico y sexual) y dimensiones latentes del maltrato (no sufre maltrato, maltrato menos severo o de menor gravedad y maltrato más severo o de mayor gravedad).

Tabla 1. Recodificación de las variables del maltrato según Delgado y colaboradores $(2006)^{18}$.

\begin{tabular}{|c|c|c|}
\hline $\begin{array}{c}\text { Preguntas } \\
\text { Su marido/pareja }\end{array}$ & Maltrato & Sí \\
\hline $\begin{array}{l}\text { 1. ¿Respecta sus } \\
\text { sentimientos?* }\end{array}$ & $\begin{array}{l}\text { nunca } \\
\text { casi nunca }\end{array}$ & $\begin{array}{l}\text { algunas veces } \\
\text { bastante veces } \\
\text { casi siempre }\end{array}$ \\
\hline $\begin{array}{l}\text { 2. ¿La ha insultado, } \\
\text { avergonzado o humillado? }\end{array}$ & $\begin{array}{l}\text { nunca } \\
\text { casi nunca }\end{array}$ & $\begin{array}{l}\text { algunas veces } \\
\text { bastante veces } \\
\text { casi siempre }\end{array}$ \\
\hline $\begin{array}{l}\text { 3. ¿Les ha amenazado a } \\
\text { usted, a sus hijos o a } \\
\text { alguna persona querida? }\end{array}$ & nunca & $\begin{array}{l}\text { casi nunca } \\
\text { algunas veces } \\
\text { bastante veces } \\
\text { casi siempre }\end{array}$ \\
\hline $\begin{array}{l}\text { 4. ¿Ha roto alguna de } \\
\text { sus cosas o algún } \\
\text { objeto de la casa? }\end{array}$ & nunca & $\begin{array}{l}\text { casi nunca } \\
\text { algunas veces } \\
\text { bastante veces } \\
\text { casi siempre }\end{array}$ \\
\hline $\begin{array}{l}\text { 5. ¿Ha intentado } \\
\text { controlar su vida o sus } \\
\text { movimientos? }\end{array}$ & $\begin{array}{l}\text { nunca } \\
\text { casi nunca }\end{array}$ & $\begin{array}{l}\text { algunas veces } \\
\text { bastante veces } \\
\text { casi siempre }\end{array}$ \\
\hline 6. ¿Le ha pegado? & nunca & $\begin{array}{l}\text { casi nunca } \\
\text { algunas veces } \\
\text { bastante veces } \\
\text { casi siempre }\end{array}$ \\
\hline $\begin{array}{l}\text { 7. ¿Ha llegado a } \\
\text { producirle heridas? }\end{array}$ & nunca & $\begin{array}{l}\text { casi nunca } \\
\text { algunas veces } \\
\text { bastante veces } \\
\text { casi siempre }\end{array}$ \\
\hline $\begin{array}{l}\text { 8. ¿La ha obligado a } \\
\text { mantener relaciones } \\
\text { sexuales sin querer } \\
\text { usted? }\end{array}$ & nunca & $\begin{array}{l}\text { casi nunca } \\
\text { algunas veces } \\
\text { bastante veces } \\
\text { casi siempre }\end{array}$ \\
\hline $\begin{array}{l}\text { 9. ¿Le ha amenazado con } \\
\text { un arma u otros } \\
\text { objetos? }\end{array}$ & nunca & $\begin{array}{l}\text { casi nunca } \\
\text { algunas veces } \\
\text { bastante veces } \\
\text { casi siempre }\end{array}$ \\
\hline $\begin{array}{l}\text { 10. ¿Ha llegado a } \\
\text { herirla con un arma u } \\
\text { otros objetos? }\end{array}$ & nunca & $\begin{array}{l}\text { casi nunca } \\
\text { algunas veces } \\
\text { bastante veces } \\
\text { casi siempre }\end{array}$ \\
\hline
\end{tabular}

${ }^{*}$ Creemos que hay una equivocación en la recodificación de esta pregunta ${ }^{18}$ (página 84). Por lo que nosotros en este estudio hemos considerado como "no maltrato", en la pregunta señalada, a las respuestas bastantes veces y casi siempre, y "sí maltrato" en nunca, casi nunca y algunas veces. 
Tabla 2. Recodificación de las variables del maltrato de acuerdo con un criterio más estrito.

\begin{tabular}{|c|c|c|}
\hline $\begin{array}{c}\text { Preguntas } \\
\text { su marido/pareja }\end{array}$ & Maltrato & Sí \\
\hline $\begin{array}{l}\text { 1. ¿Respecta sus } \\
\text { sentimientos? }\end{array}$ & $\begin{array}{l}\text { bastante veces } \\
\text { casi siempre }\end{array}$ & $\begin{array}{l}\text { nunca } \\
\text { casi nunca } \\
\text { algunas veces }\end{array}$ \\
\hline $\begin{array}{l}\text { 2. ¿La ha insultado, } \\
\text { avergonzado o humillado? }\end{array}$ & nunca & $\begin{array}{l}\text { casi nunca } \\
\text { algunas veces } \\
\text { bastante veces } \\
\text { casi siempre }\end{array}$ \\
\hline $\begin{array}{l}\text { 3. ¿Les ha amenazado a } \\
\text { usted, a sus hijos o a } \\
\text { alguna persona querida? }\end{array}$ & nunca & $\begin{array}{l}\text { casi nunca } \\
\text { algunas veces } \\
\text { bastante veces } \\
\text { casi siempre }\end{array}$ \\
\hline $\begin{array}{l}\text { 4. ¿Ha roto alguna de } \\
\text { sus cosas o algún } \\
\text { objeto de la casa? }\end{array}$ & nunca & $\begin{array}{l}\text { casi nunca } \\
\text { algunas veces } \\
\text { bastante veces } \\
\text { casi siempre }\end{array}$ \\
\hline $\begin{array}{l}\text { 5. ¿Ha intentado } \\
\text { controlar su vida o sus } \\
\text { movimientos? }\end{array}$ & nunca & $\begin{array}{l}\text { casi nunca } \\
\text { algunas veces } \\
\text { bastante veces } \\
\text { casi siempre }\end{array}$ \\
\hline 6. ¿Le ha pegado? & nunca & $\begin{array}{l}\text { casi nunca } \\
\text { algunas veces } \\
\text { bastante veces } \\
\text { casi siempre }\end{array}$ \\
\hline $\begin{array}{l}\text { 7. ¿Ha llegado a } \\
\text { producirle heridas? }\end{array}$ & nunca & $\begin{array}{l}\text { casi nunca } \\
\text { algunas veces } \\
\text { bastante veces } \\
\text { casi siempre }\end{array}$ \\
\hline $\begin{array}{l}\text { 8. ¿La ha obligado a } \\
\text { mantener relaciones } \\
\text { sexuales sin querer } \\
\text { usted? }\end{array}$ & nunca & $\begin{array}{l}\text { casi nunca } \\
\text { algunas veces } \\
\text { bastante veces } \\
\text { casi siempre }\end{array}$ \\
\hline $\begin{array}{l}\text { 9. ¿Le ha amenazado con } \\
\text { un arma u otros } \\
\text { objetos? }\end{array}$ & nunca & $\begin{array}{l}\text { casi nunca } \\
\text { algunas veces } \\
\text { bastante veces } \\
\text { casi siempre }\end{array}$ \\
\hline $\begin{array}{l}\text { 10. ¿Ha llegado a } \\
\text { herirla con un arma u } \\
\text { otros objetos? }\end{array}$ & nunca & $\begin{array}{l}\text { casi nunca } \\
\text { algunas veces } \\
\text { bastante veces } \\
\text { casi siempre }\end{array}$ \\
\hline
\end{tabular}

Los miembros del grupo de investigación se desplazaron a los centros asistenciales para explicar el procedimiento de distribución con el mismo criterio. Fue negociado con las direcciones de los centros un lugar accesible, a la vez que seguro, para la ubicación de una urna preparada al efecto que respetase en todo momento la intimidad. El cuestionario distribuido de forma aleatoria se entregaba acompañado de un sobre que permitía devolverlo 
cerrado de forma anónima y confidencial. El plazo de recogida de las urnas fue de 7 a 10 días y era realizado por miembros del grupo investigador.

\section{Análisis estadístico}

Descripción de variables cualitativas recuento y error estándar del porcentaje y para las cuantitativas media, desviación típica y error estándar de la media.

Para la codificación, recodificación y análisis de los datos se utilizó el programa estadístico SPSS (versión 14.0 para Windows)

\section{RESULTADOS}

Se distribuyeron 260 sobres con cuestionario, y como ya se ha señalado, casi el triple necesario para la muestra, debido a la perspectiva de falta de respuesta y principalmente por respeto extremo a la intimidad. De estos, $117(45,0 \%)$ no fueron depositados en las cajas, y de los 143 cuestionarios recogidos se cumplimentaron 140 por lo tanto la frecuencia de respuestas fue $54,0 \%$. De los 140 cuestionarios se excluyeron 37 por estar incompletos y 1 que no había tenido pareja, la frecuencia total de respuestas fue de $39,6 \%$.

En la tabla 3 se presentan las características sociodemográficas de las enfermeras del estudio. El 98,0\% eran de la Provincia de Córdoba, el 82,4\% estaban casadas y el 53,9\% convivían con el marido o pareja. La edad media era de 40,6 años (24-57); el número medio de hijos y/o personas mayores dependientes a cargo de la participante en el estudio era de 2,1 y la media de años de relación con la pareja de 17,2. El 90,2\% trabajaban en centros sanitarios públicos, en el $56,9 \%$ el ámbito de trabajo era urbano y la mayoría de las enfermeras $(89,2 \%)$ ocupaban el puesto de enfermera base. El 85,3\% tenían un sustento económico familiar con ambos sueldos (de la enfermera y el de la pareja). El $24,5 \%$ de las parejas pertenecía a la clase social I.

Tabla 3. Características sociodemográficas de las enfermeras que prestan sus servicios en la Provincia de Córdoba $(n=102)$.

\begin{tabular}{|c|c|}
\hline Variables & n (\%) \\
\hline \multicolumn{2}{|l|}{ Provincia de origen } \\
\hline Cádiz & $1(1,0)$ \\
\hline Córdoba & $100(98,0)$ \\
\hline Jaén & $1(1,0)$ \\
\hline \multicolumn{2}{|l|}{ Estado civil } \\
\hline Soltera & $11(10,8)$ \\
\hline Casada/pareja estable & $84 \quad(82,4)$ \\
\hline Separada/divorciada & $6(5,9)$ \\
\hline Viuda & $1(1,0)$ \\
\hline \multicolumn{2}{|l|}{ Convivencia } \\
\hline Marido/pareja ${ }^{a}$ & $55(53,9)$ \\
\hline Familia origen/familia parejab & $16(15,7)$ \\
\hline Sola & $1(1,0)$ \\
\hline $\operatorname{Hijo(s)^{c}}$ & $5 \quad(4,9)$ \\
\hline$a+c$ & $22(21,6)$ \\
\hline$a+b+c$ & $3(2,9)$ \\
\hline Centro de trabajo & \\
\hline Público & $92(90,2)$ \\
\hline
\end{tabular}




\begin{tabular}{|c|c|c|}
\hline Privado & 10 & $(9,8)$ \\
\hline \multicolumn{3}{|l|}{ Ámbito de trabajo } \\
\hline Rural & 44 & $(43,1)$ \\
\hline Urbano & 58 & $(56,9)$ \\
\hline \multicolumn{3}{|l|}{ Puesto que ocupa en el trabajo } \\
\hline Enfermera base & 91 & $(89,2)$ \\
\hline Gestión & 11 & $(10,8)$ \\
\hline \multicolumn{3}{|l|}{ Sustento económico familiar } \\
\hline Su sueldo & 11 & $(10,8)$ \\
\hline Sueldo pareja & 4 & $(3,9)$ \\
\hline Ambos sueldos & 87 & $(85,3)$ \\
\hline \multicolumn{3}{|l|}{ Clase social de la pareja* } \\
\hline Clase I & 25 & $(24,5)$ \\
\hline Clase II & 20 & $(19,6)$ \\
\hline Clase IIIa & 15 & $(14,7)$ \\
\hline Clase IIIb & 22 & $(21,6)$ \\
\hline Clase IIIC & 1 & $(1,0)$ \\
\hline Clase IVa & 15 & $(14,7)$ \\
\hline Clase IVb & 2 & $(2,0)$ \\
\hline Clase V & 2 & $(2,0)$ \\
\hline \multicolumn{3}{|l|}{ Hijos y/o personas mayores dependientes } \\
\hline Sí & 29 & $(28,4)$ \\
\hline No & 73 & $(71,6)$ \\
\hline Número de dependientes, media (DE) & 2,1 & $(1,0)$ \\
\hline Años de relación con la pareja, media (DE) & 17,2 & $(9,1)$ \\
\hline Edad de la enfermera (años), media (DE) & 40,6 & $(8,4)$ \\
\hline
\end{tabular}

${ }^{*}$ Clasificación de clase social según la Sociedad Española de Epidemiología ${ }^{19}$. I = Directivos de la Administración pública y de empresas de 10 o más asalariados, profesiones asociadas con titulaciones de segundo y tercer ciclo universitario. II = Directivos de empresas con menos de 10 asalariados. Profesiones asociadas con una titulación de primer ciclo universitario. Técnicos y profesionales de apoyo. Artistas y deportistas. Illa $=$ Personal de tipo administrativo y profesionales de apoyo a la gestión administrativa y financiera. Trabajadores de los servicios personales y de seguridad. Illb $=$ Trabajadores por cuenta ajena. IIIc $=$ Supervisores de trabajadores manuales. IVa $=$ Trabajadores manuales cualificados. $\mathbf{I V b}=$ Trabajadores manuales semicualificados. $\mathbf{V}=$ Trabajadores no cualificados. $\mathbf{D E}=$ desviación típica.

Se analizaron los datos del maltrato considerando la subjetividad de la comprensión de violencia, especialmente el maltrato psíquico debido a su carácter polisémico. Las recodificaciones utilizadas fueron, una la utilizada por Delgado y colaboradores $(2006)^{18}$ (tabla 1) y otra más estricta (tabla 2). La intención es que cada lector se decida por una u otra recodificación según su criterio personal.

En la tabla 4 se observa la frecuencia absoluta y el porcentaje del cuestionario de malos tratos. Sólo las preguntas 2 y 5 presentaron resultados distintos en ambas recodificaciones: en la pregunta 2, hay maltrato en $10,8 \%$ (criterio más permisivo) y en $27,5 \%$ (criterio más estricto), en cuanto a la pregunta 5 , el $16,7 \%$ (criterio más permisivo) y el $35,3 \%$ (criterio más estricto) presentaron maltrato. 
Tabla 4. Frecuencia absoluta y porcentaje de las preguntas de mal trato en la muestra enfermeras que prestan sus servicios en la Provincia de Córdoba $(n=102)$.

\begin{tabular}{|c|c|c|c|c|}
\hline \multirow[t]{2}{*}{$\begin{array}{c}\text { Preguntas } \\
\text { Su marido/pareja }\end{array}$} & \multicolumn{2}{|c|}{$\begin{array}{c}\text { Maltrato con } \\
\text { criterio más } \\
\text { permisivo (Delgado } \\
\text { y cols., 2006) }\end{array}$} & \multicolumn{2}{|c|}{$\begin{array}{l}\text { Maltrato con } \\
\text { criterio más } \\
\text { estrito }\end{array}$} \\
\hline & $\begin{array}{ll} & \text { No } \\
\text { n } \quad\left(\frac{\circ}{0}\right)\end{array}$ & $\begin{array}{l}S i \\
n(\%)\end{array}$ & $\mathrm{n}\left(\frac{\circ}{0}\right)^{\text {No }}$ & $\begin{array}{c}S i ́ \\
\mathrm{n}\left(\frac{\circ}{0}\right)\end{array}$ \\
\hline $\begin{array}{l}\text { 1. ¿Respecta sus } \\
\text { sentimientos? }\end{array}$ & $94(92,2)$ & $8(7,8)$ & $94(92,2)$ & $8(7,8)$ \\
\hline $\begin{array}{l}\text { 2. ¿la ha } \\
\text { insultado, } \\
\text { avergonzado o } \\
\text { humillado? }\end{array}$ & $91(89,2)$ & $11(10,8)$ & $74(72,5)$ & $28(27,5)$ \\
\hline $\begin{array}{l}\text { 3. ¿Les ha } \\
\text { amenazado a usted, } \\
\text { a sus hijos o a } \\
\text { alguna persona } \\
\text { querida? }\end{array}$ & $91(89,2)$ & $11(10,8)$ & $91(89,2)$ & $11(10,8)$ \\
\hline $\begin{array}{l}\text { 4. ¿Ha roto alguna } \\
\text { de sus cosas o } \\
\text { algún objeto de } \\
\text { la casa? }\end{array}$ & $90(88,2)$ & $12(11,8)$ & $90(88,2)$ & $12(11,8)$ \\
\hline $\begin{array}{l}\text { 5. ¿Ha intentado } \\
\text { controlar su vida } \\
\text { o sus movimientos? }\end{array}$ & $85(83,3)$ & $17(16,7)$ & $66(64,7)$ & $36(35,3)$ \\
\hline 6. ¿Le ha pegado? & $99(97,1)$ & $3(2,9)$ & $99(97,1)$ & $3(2,9)$ \\
\hline $\begin{array}{l}\text { 7. ¿Ha llegado a } \\
\text { producirle } \\
\text { heridas? }\end{array}$ & $101(99,0)$ & $1(1,0)$ & $101(99,0)$ & $1(1,0)$ \\
\hline $\begin{array}{l}\text { 8. ¿La ha obligado } \\
\text { a mantener } \\
\text { relaciones } \\
\text { sexuales sin } \\
\text { querer usted? }\end{array}$ & $96(6,0)$ & $6(5,9)$ & $96(6,0)$ & $6(5,9)$ \\
\hline $\begin{array}{l}\text { 9. ¿Le ha } \\
\text { amenazado con un } \\
\text { arma u otros } \\
\text { objetos? }\end{array}$ & $100(98,0)$ & $2(2,0)$ & $100(98,0)$ & $2(2,0)$ \\
\hline $\begin{array}{l}\text { 10. ¿Ha llegado a } \\
\text { herirla con un } \\
\text { arma u otros } \\
\text { objetos? }\end{array}$ & $101(99,0)$ & $1(1,0)$ & $101(99,0)$ & $1(1,0)$ \\
\hline
\end{tabular}

Los datos expresan la frecuencia absoluta (porcentaje).

La figura 1 indica los resultados del tipo de maltrato de acuerdo con un criterio más estricto y según la recodificación de Delgado y cols (2006) ${ }^{18}$ respectivamente: el $52,9 \%$ y $74,5 \%$ de las enfermeras estudiadas no sufren ningún tipo de maltrato, el 41,2\% y 19,6\% presenta maltrato psicológico exclusivo, seguido de maltratos asociados: los tres tipos juntos 
(psicológico, físico y sexual) y psicológico acompañado del sexual ambos con $2,9 \%$ en las dos recodificaciones. Tampoco hubo en las demás asociaciones maltratos físicos y sexuales exclusivos.

Figura 1: Ausencia de maltrato y su presencia de acuerdo con los tipos encontrados en la muestra de las enfermeras que prestan sus servicios en la Provincia de Córdoba $(n=102)$.

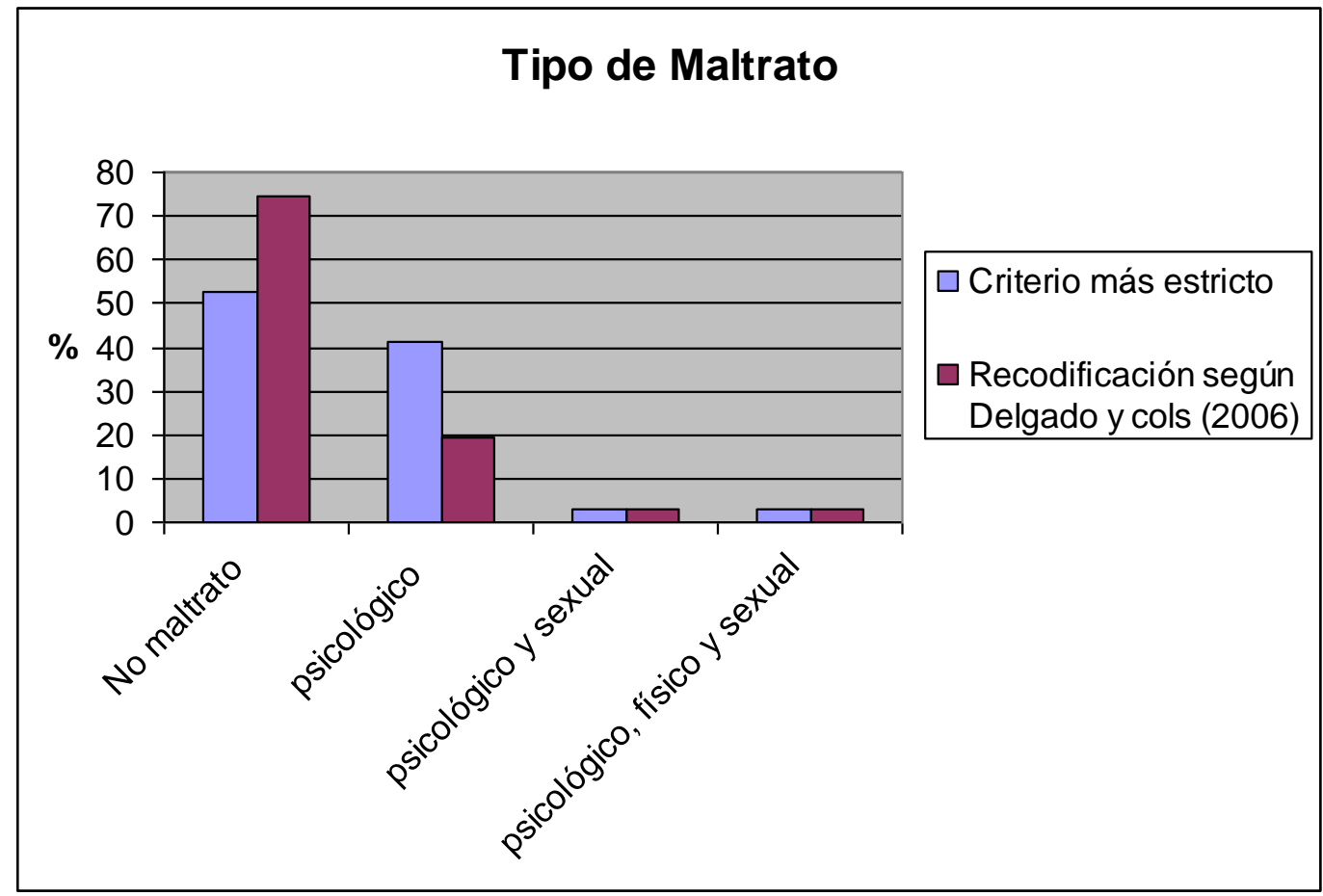

La figura 2 muestra las dimensiones latentes del maltrato en la muestra de las enfermeras que prestan sus servicios en la Provincia de Córdoba. El 11,8\% presentó maltrato más severo o de mayor gravedad en ambas recodificaciones.

Figura 2: Dimensiones latentes del maltrato en la muestra de las enfermeras que prestan sus servicios en la Provincia de Córdoba $(n=102)$.

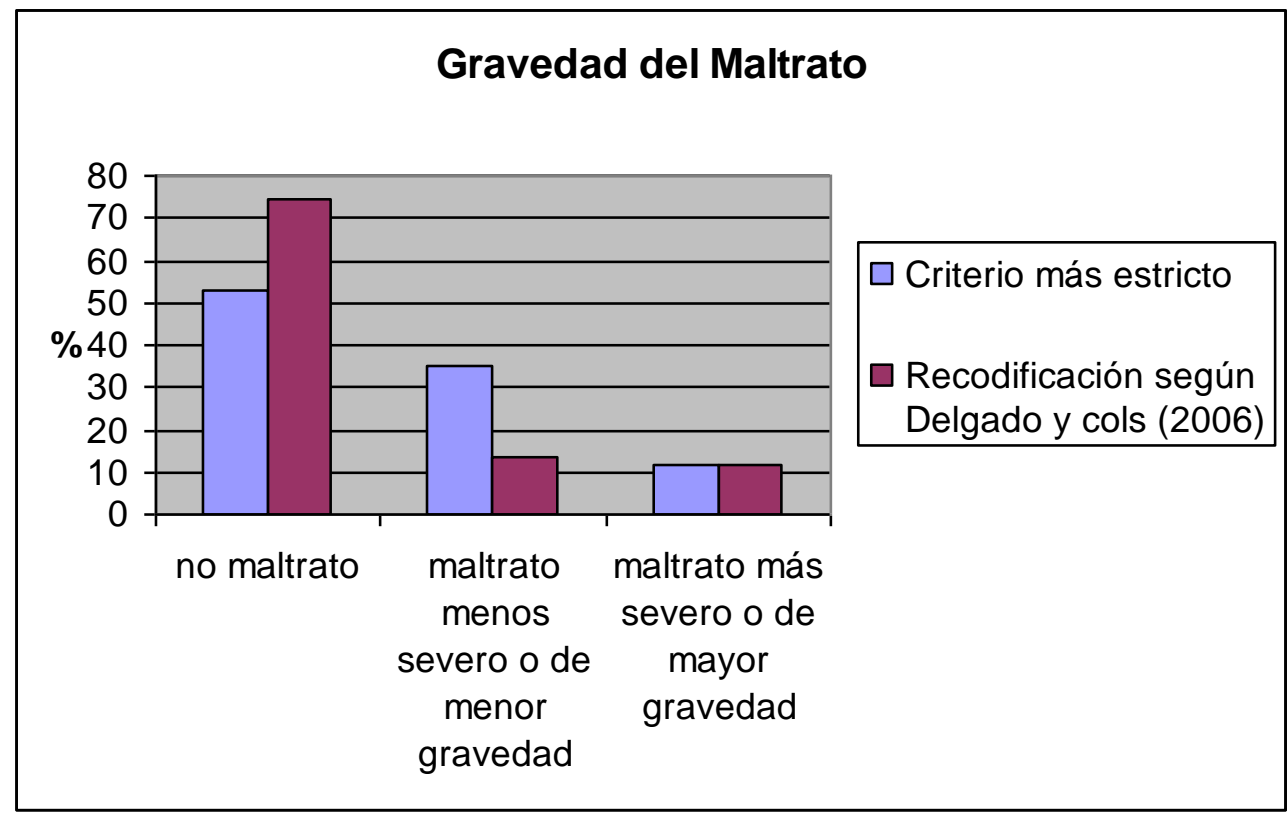


En términos generales y con los datos obtenidos en este colectivo de mujeres enfermeras la prevalencia de maltrato se sitúa en el $26 \%$.

\section{DISCUSIÓN}

El presente trabajo es el primero en España a tratar el tema "Violencia por compañero íntimo - VCl - contra las mujeres enfermeras que desarrollan sus actividades profesionales en los sistemas de salud".

Como limitaciones del estudio señalamos que tiene los sesgos habituales de los estudios autorreferidos. Además es posible que haya una frecuente ocultación de hechos de violencia por la intimidad del tema y/o por vergüenza. En cualquier caso, todos estos hechos pueden llevar a infraestimar los datos del real maltrato. Se deben hacer con precaución todas las comparaciones de los resultados obtenidos del mismo con los observados en otros trabajos, dada las diversas naturalezas del maltrato y de los instrumentos utilizados.

En relación a la subjetividad de definición de violencia, principalmente la psicológica, puede asumir distintos significados dependiendo de la cultura de un determinado grupo social ${ }^{20}$.

En el presente estudio se indica que la VCl contra enfermeras que trabajan en la provincia de Córdoba puede tener las siguientes expresiones: violencia psicológica exclusiva, seguida de por los tres tipos juntos y por la psicológica acompañada de la sexual.

Entre las profesionales estudiadas, la presencia de maltrato psicológico $(41,2 \%$ - criterio más estricto - y $19,6 \%$ - criterio más permisivo) en ambas recodificaciones fue predominante. Estos datos se ajustan con la literatura en el sentido de que las formas exclusivas de violencia física y sexual son más raras de ser encontradas, en comparación con la violencia psicológica exclusiva, más frecuente ${ }^{21-24}$. En el estudio realizado por Coker y colaboradores $(2002)^{25}$ la violencia psicológica intensa tenía una asociación mayor con síntomas depresivos en las mujeres maltratadas que la violencia física.

Un estudio canadiense ${ }^{26}$, identificó que $38,0 \%$ de las enfermeras ya habían sufrido algún tipo de $\mathrm{VCl}$ y que la violencia psicológica $(26,9 \%)$ fue superior a la física $(14,6 \%)$ y también a la sexual $(8,1 \%)$. Se resaltó que la frecuencia de $\mathrm{VCl}$ entre las enfermeras estudiadas superó la vivencia de tal violencia entre mujeres canadienses de la población general $(29,0 \%)$. Lo mismo ocurrió con las enfermeras de la muestra de Córdoba $(26,0 \%)$ en relación a la población general de mujeres españolas (12,0\%).

Otro estudio en Brasil con enfermeras paulistas mostró una frecuencia mayor que el anterior para la violencia psicológica $(45,0 \%)^{27}$.

La asociación de las violencias psicológica, física y sexual se constituye en una situación extremadamente grave y en el presente estudio fue encontrada en 3 enfermeras $(2,9 \%)$.

A partir del análisis factorial exploratorio realizado por Delgado y colaboradores $(2006)^{18}$ se reveló la presencia de un constructo empírico en las mujeres estudiadas. La primera dimensión, maltrato menos severo o de menor gravedad, incluye las preguntas: 1, 2, 3, 5, 6 y 8. La segunda dimensión empírica, el maltrato más severo o de mayor gravedad, se manifiesta en las preguntas 4, 7, 9 y 10. La presencia de este tipo de maltrato en 11,8\% (ambas recodificaciones), de las enfermeras estudiadas, nos lleva a sugerir que se debería valorar la existencia de un riesgo potencial para la integridad de estas mujeres. 
El maltrato sexual exclusivo es un hallazgo poco frecuente en la mayoría de los países. En este estudio tres casos (2,9\%) presentaban violencia sexual acompañados de violencia psicológica. No hubo caso de violencia sexual o física exclusivas. Muchos trabajos hacen referencia a los maltratos físicos y sexuales, ya que son los que poseen mayor evidencia ${ }^{28}$. Mientras tanto, la violencia psicológica es la más frecuente y puede tener un gran impacto sobre la salud como las otras formas de maltrato ${ }^{29,30}$.

En conclusión, establecida la existencia de malos tratos en las mujeres enfermeras de la muestra de Córdoba se constata que las manifestaciones van referidas al aspecto psicológico. Estos resultados recomiendan establecer acciones encaminadas a conocer cómo la mujer enfermera, que sufre $\mathrm{VCl}$, afronta la atención a mujeres que sufren el mismo problema, así como a la atención de estas profesionales.

\section{FINANCIACIÓN}

Este estudio está siendo financiado por la Consejería de Salud de la Junta de Andalucía (PI0109/2008).

\section{BIBLIOGRAFÍA}

1. United Nations - UN. Fourth World Conference on Women. In: Violence against Women Diagnosis. Beijing; $1995 . \quad$ Disponible en: http://www.un.org/womenwatch/daw/beijing/platform/violence.htm\#diagnosis Consultado: 17 oct. 2008.

2. Organización Mundial de la Salud - OMS. Violencia contra la mujer: Consulta de la OMS, Ginebra, 5-7 febrero de 1996. Ginebra. 1996. Documento FRH/WHD/96.27. Disponible en: http://whqlibdoc.who.int/hq/1996/FRH WHD 96.27.pdf, desde el 18 de marzo de 2005). Consultado: 04 abr. 2008.

3. Santos SF. Saúde da mulher e direitos reprodutivos. Recife: Fundação Ford; 2001.

4. World Report on violence and Health. Geneva: World Health Organization; 2002. Disponible en: http://whqlibdoc.who.int/publications/2002/9241545615 eng.pdf Consultado: 10 nov. 2008.

5. García-Moreno C. Violencia contra la mujer. Género y equidad en la salud. Organización Panamericana de la Salud y Harvard Center for population and Development Studies; 2000. Disponible en: http://www.paho.org/Spanish/DBl/po06.htm Consultado: 10 nov. 2008.

6. Heise L, Pitanguy J, Germain A. Violence against women. The hidden health burden. Washignton (DC): World Bank; 1994. (World Bank Discussion Papers, 255.

7. Alberdi I, MarcosLR. Violencia: Tolerancia Cero. Barcelona: Fundación "La Caixa"; 2005.

8. Richardson J, Coid J, Petruckevitch A, Chung WS, Moorey S, Feder G. identifying domestic violence: cross sectional study in primary Care. BMJ 2002;324:1-6.

9. Bradley F, Smith M, Long J, O'Dowd T. Reported frequency of domestic violence: cross sectional survey of women attending general practice. BMJ 2002;324:271-4.

10. Jacobson A, Richardson B. Assault experiences of 100 psychiatric inpatients: evidence of the need for routine inquiry. Am J Psychiatry 1987;144:908-12.

11. Thomas, Telmon, Alleery, Pauwels, Rougé. La violence conjugale dix ans après. Le Concours Médical 2000;122:2041-4.

12. Campbell JC. Child abuse and wife abuse: the connections: Med Med J 1994;43:349-50. 
13. Hotch D, Grunfeld AF, Mackay K, Cowan L. An emergency department -based domestic violence intervention program: findings after one year. J Emerg Med 1996;14:111-7.

14. Blanco P, Ruiz-Jarabo C, Vinuesa LG, Martín-García M. La violencia de pareja y la salud de las mujeres. Gac Sanit 2004;18(Supl 1):182-8.

15. Goldberg WG, Tomlanovich MC. Domestic violence victims in the emergency department: new findings. JAMA 1984;251:3259-64.

16. Coll-Vinent B, Echeverría T, Farràs Ú, Rodríguez D, Millá J, Santiñà M. El personal sanitario no percibe la violencia doméstica como un problema de salud. Gac Sanit. 2008; 22(1):7-10.

17. Instituto de la Mujer. Macroencuesta: "Violencia contra las mujeres". Porcentaje de mujeres maltratadas, según CC.AA. 1999. Disponible en: http://www.migualdad.es/mujer/mujeres/cifras/violencia/macroencuesta violencia.ht m Consultado: 04 abr. 2008.

18. Delgado A, Aguar M, Castellano M, Luna del Castillo JD. Validación de una escala para la medición de los malos tratos a mujeres. Atención primaria 2006, 38 (2): 82 89.

19. Álvarez-Dardet C, Alonso J, Domingo A, Regidor E. Grupo de trabajo de la Sociedad Española de Epidemiología. La medición de la clase social en ciencias de la salud. Barcelona: G Editores; 1995.

20. Krug EG, Dahlber LL, Merc JA, Zwi AB, Lozano R. [WHO] World Health Organization. Report on Violence and Health, Chapter 1 y 4, Violence: a global public health problem and violence y Violence by partner. Genebra; 2002. p.121/90-121.

21. Harwell TS, Moore KR, Spence MR. Physical violence, intimate partner violence, and emotional abuse among adult American Indian men and women in Montana. Prev Med. 2003;37:297-303.

22. Kronbauer JFD, Meneghel SN. Perfil da violencia de gênero perpetrada por companheiro. Rev Saúde Pública. 2005; 39 (5):695-701.

23. Ruiz-Pérez I, Plazaola-Castaño J, Blanco-Prieto P, González-Barranco JM, AyusoMartín P, Montero-Piñar MI, Grupo de Estudios para la Violencia de Género. Gac Sanit. 2006;20(3):202-8.

24. Schraiber LB, D'Oliveira AFPL, França-Junior I, Diniz S, Portella AP, Ludermir AB, Valença O, Couto MT. Prevalência da violência contra a mulher por companheiro íntimo em regiões do Brasil. Rev Saúde Pública. 2007;41(5):797-807.

25. Coker AL, Davis KE, Arias I. Physical and mental health effects of intimate partner violence for men and women. Am J Prev Med. 2002;23:260-8.

26. Jansen PA, Basso MC, Costanzo RB. The prevalence of domestic violence among obstretic nurses. Womens Health Issues. 1998;8(5):317-23.

27. Oliveira AR. Violência de gênero contra mulheres profissionais de enfermagem de um hospital geral do município de São Paulo. [Dissertação] Disponible en: http://www.teses.usp.br/teses/disponiveis/5/5137/tde-10122007-221036/ Consultado: 29 abr. 2008.

28. Krug E, Dahlberg L, Mercy J, Zwi A, Lozano R, editors. World report on violence and health. Genève: World Health Organization; 2002.

29. Pico-Alfonso MA, García-Linares Ml, Celda-Navarro N, Herbet J, Martínez M. Changes in cortisol and dehydroepiandrosterone in women victims of physical and psychological intimate partner violence. Biol Psychiatry. 2004;66:965-42.

30. García-Linares MI, Sánchez-Lorente S, Coe CL, Martínez M. Intimate male partner violence impairs immune control over herpes simples virus type 1 in physically and psychologically abused women. Psychosom Med. 2004;66:965-72. 
